\title{
ORNLTM-12978
}

OAK RIDGE NATIONAL LABORATORY

MARTIN MARUETTA

\section{The Least Squares Fit of a Hyperplane to Uncertain Data}

MANAGED BY

MARTIN MARIETTA ENERGY SYSTEMS, INC.

FOR THE UNITED STATES

DEPARTMENT OF ENERGY

D. B. Reister 
This report has been reproduced directly from the best available copy.

Available to DOE and DOE contractors from the Office of Scientific and Technical Information, P.O. Box 62, Oak Ridge, TN 37831; prices available from (615) 576-8401, FTS 626-8401.

Available to the public from the National Technical Information Service, U.S. Department of Commerce, 5285 Port Royal Rd., Springfield, VA 22161.

This report was prepared as an account of work sponsored by an agency of the United States Government. Neither the United States Government nor any agency thereof, nor any of their employees, makes any warranty, express or implied, or assumes any legal liability or responsibility for the accuracy, completeness, or usefulness of any information, apparatus, product, or process disclosed, or represents that its use would not infringe privately owned rights. Reference herein to any specific commercial product, process, or service by trade name, trademark, manufacturer, or othenwise, does not necessarily constitute or imply its endorsement, recommendation, or favoring by the United States Government or any agency thereof. The views and opinions of authors expressed herein do not necessarily state or reflect those of the United States Government or any agency thereof. 


\section{DISCLAIMER}

Portions of this document may be illegible in electronic image products. Images are produced from the best available original document. 
Computer Science and Mathematics Division

\title{
THE LEAST SQUARES FIT OF A HYPERPLANE TO UNCERTAIN DATA
}

\author{
D. B. Reister
}

\section{DATE PUBLISHED - MAY 1995}

\author{
Prepared by \\ Oak Ridge National Laboratory \\ Oak Ridge, Tennessee \\ managed by \\ MARTIN MARIETTA ENERGY SYSTEMS, INC. \\ for the \\ U.S. DEPARTMENT OF ENERGY \\ under Contract No. DE-AC05-84OR21400
}




\section{TABLE OF CONTENTS}

LIST OF TABLES $\ldots \ldots \ldots \ldots \ldots \ldots \ldots \ldots \ldots \ldots \ldots \ldots \ldots \ldots, \ldots \ldots$

ACKNOWLEDGMENTS $\ldots \ldots \ldots \ldots \ldots \ldots \ldots \ldots \ldots \ldots \ldots \ldots$ vii ABSTRACT $\ldots \ldots \ldots \ldots \ldots \ldots \ldots \ldots \ldots \ldots \ldots \ldots \ldots \ldots \ldots \ldots \ldots \ldots \ldots \ldots$

1. INTRODUCTION $\ldots \ldots \ldots \ldots \ldots \ldots \ldots \ldots \ldots \ldots \ldots \ldots \ldots \ldots \ldots$ 1

2. LEAST SQUARES ESTIMATION OF A HYPERPLANE $\ldots \ldots \ldots \ldots 3$

3. NUMERICAL EXPERIMENTS $\ldots \ldots \ldots \ldots \ldots \ldots \ldots \ldots \ldots \ldots$

4. CONCLUSIONS $\ldots \ldots \ldots \ldots \ldots \ldots \ldots \ldots \ldots \ldots \ldots \ldots \ldots \ldots \ldots$

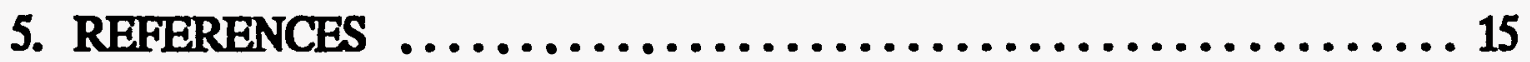




\section{LIST OF TABLES}

Tables

Pages

1. The error measure for synthetic data set for four methods $\ldots \ldots \ldots \ldots 12$

2. The least squares estimates of parameters $a$ and $d$ for five synthetic data sets . ................................ 12 


\section{ACKNOWLEDGMENTS}

After reading reference 2, E. M. Oblow encouraged the author to find the exact (rather than approximate) solution to this problem. This research was performed under sponsorship of the Engineering Research Program, Office of Basic Energy Sciences, of the U. S. Department of Energy, under contract DE-AC05-84OR21400 with Martin Marietta Energy Systems, Inc. 


$$
\text { - } \ldots \text { - }
$$$$
\ldots
$$ 


\begin{abstract}
For many least squares problems, the uncertainty is in one of the variables [for example, $y=f(x)$ or $z=f(x, y)$ ]. However, for some problems, the uncertainty is in the geometric transformation from measured data to Cartesian coordinates and all of the calculated variables are uncertain.

We have formulated a constrained optimization problem to determine the least squares fit of a hyperplane to uncertain data. The first order necessary conditions require the solution of an eigenvalue problem. We have shown that the solution satisfies the second order conditions (the Hessian matrix is positive definite). Thus, our solution satisfies the sufficient conditions for a local minimum. We have performed numerical experiments that demonstrate that our solution is superior to alternative methods.
\end{abstract}




\section{INTRODUCTION}

We became interested in this problem because we wanted to calibrate our laser range cameras. Laser range cameras are powerful tools for defining the Cartesian coordinates of surfaces of objects in an environment. The camera scans a laser beam over a scene and determines the distance $(r)$ by the time required for the beam to reach an object and return. The beam is directed back and forth $(\phi)$ and up and down $(\theta)$ by two rotating mirrors. Thus, the data measured by the camera has the form $(r, \phi, \theta)$. The geometry of the camera can be used to map the measured data to Cartesian coordinates $(x, y, z)$.

Since the geometry of the camera may not be known precisely, the cameras can be calibrated by comparing the calculated surface shapes to the known surface shapes. The most simple surface is a plane and many physical objects have planar surfaces. Thus, an important problem in the calibration of range cameras is to find the best (least squares) fit of a plane to a set of $3 \mathrm{D}$ points.

For many least squares problems, the uncertainty is in one of the variables [for example, $y=f(x)$ or $z=f(x, y)]$. However, for this problem, the uncertainty is in the geometric transformation from measured data to Cartesian coordinates and all three of the calculated variables are uncertain.

We are all familiar with the linear regression problem of determining the least squares fit of data to a straight line $(y=a+b x)$. Two linear equations (the normal equations) are solved to determine the parameters $(a$ and $b$ ). For the general case with $M$ basis functions, the $M$ parameters are determined by solving the $M$ normal equations. The book by Lawson [1] provides a good introduction to the methods for solving least squares problems.

When both $x$ and $y$ are uncertain, a line is determined by three parameters $(a+b x+c y=0)$. To minimize the sum of the squares of the errors, the three parameters satisfy a system of three homogeneous equations $[D s=0$ where $\left.s^{T}=(a, b, c)\right]$. For the general case of a hyperplane in an $N$ dimensional space, the parameters satisfy a system of $N+1$ homogeneous equations. The homogeneous equations will have a zero solution unless the matrix $D$ is singular.

In a recent paper [2], we estimated an approximate solution of the homogeneous equations by adding a small pertubation to make the matrix $D$ singular and by solving the resultant eigenvalue problem [ $D s=\gamma s]$. The approximate solution was compared to the set of solutions obtained by neglecting one of the homogeneous equations at a time. Pratt [3] estimates the solution of the homogeneous equations by neglecting 
the last row of the Cholesky decomposition of the matrix $D$. Nievergelt[4] has followed a different path to derive the same algorithm that we shall develop in this paper.

In the next section, we will formulate a constrained optimization problem to determine the least squares fit of a hyperplane to uncertain data. The first order necessary conditions require the solution of an eigenvalue problem. We will show that the solution satisfies the second order conditions (the Hessian matrix is positive definite). Thus, our solution satisfies the sufficient conditions for a local minimum. In the third section, we will discuss the results of numerical experiments that demonstrate that our solution is superior to both our previous method and to Pratt's method. 


\section{LEAST SQUARES ESTIMATION OF A HYPERPLANE}

Our motivation was to fit a plane to three dimensional range data. We will generalize our problem from three dimensions to $N$ dimensions. We assume that we receive noisy measurements of points $(p)$ in an $N$ dimensional space:

$$
p=\left(x_{1}, x_{2}, \cdots, x_{N}\right)
$$

The data will be a set of points $\left(p_{j}\right)$, where $j$ ranges from 1 to $M$ :

$$
p_{j}=\left(x_{1 j}, x_{2 j}, \ldots, x_{N j}\right)
$$

A hyperplane is defined by the following equation:

$$
d+\sum_{i=i}^{N} a_{i} x_{i}=0
$$

If the parameter vector $\mathbf{a}$ is a unit vector, $d$ is the perpendicular distance from the origin to the plane. We will require that the parameter vector a be a unit vector:

$$
\sum_{i=1}^{N}\left(a_{i}\right)^{2}=1
$$

Since the data will not all lie on the hyperplane, we can define an error $\left(e_{j}\right)$ for each point:

$$
e_{j}=d+\sum_{i=i}^{N} a_{i} x_{i j}
$$

We will choose the parameters (the $N$ vector $a$ and the scalar $d$ ) to minimize the weighted sum of the squares of the errors subject to the constraint that $a$ is a unit 
vector. We use a Lagrange multiplier $\lambda$ for the constraint and define the Lagrangian function $L$ by:

$$
L=\sum_{j=1}^{M} w_{j}\left(e_{j}\right)^{2}+\lambda\left[1-\sum_{i=1}^{N}\left(a_{i}\right)^{2}\right]
$$

where the weights $\left(w_{j}\right)$ are nonnegative and sum to 1 .

Using Eq. (5), $L$ may be written:

$$
L=d^{2}+2 d \sum_{i=1}^{N} a_{i} B_{i}+\sum_{i=1}^{N} \sum_{k=1}^{N} a_{j} a_{k} A_{i k}+\lambda\left[1-\sum_{i=1}^{N}\left(a_{i}\right)^{2}\right]
$$

where:

$$
\begin{aligned}
A_{i k} & =\sum_{j=1}^{M} w_{j} x_{i j} x_{k j} \\
B_{i} & =\sum_{j=i}^{M} w_{j} x_{i j}
\end{aligned}
$$

The A matrix is symmetric $\left(A_{i k}=A_{t i}\right)$.

The first order necessary conditions for the parameters that minimize $L$ is that the partial derivatives of $L$ with respect to each parameter are zero:

$$
d+\sum_{i=1}^{N} a_{i} B_{i}=0
$$




$$
d B_{k}+\sum_{i=1}^{N} a_{i} A_{i k}-\lambda a_{k}=0, \text { for } k=1 \text { to } N
$$

Nievergelt[4] shows that the hyperplane must pass through the centroid of the data. Equation (10) requires that the vector $B$ be a point on the hyperplane, while Eq. (9) defines the vector $B$ to be the centroid of the data. Thus, Eq. (10) supports Nievergelt's conclusion.

We can use Eq. (10) to eliminate the parameter $d$ from Eq. (11):

$$
-\sum_{i=1}^{N} a_{i} B_{i} B_{k}+\sum_{i=1}^{N} a_{i} A_{i k}-\lambda a_{k}=0, \text { for } k=1 \text { to } N
$$

Thus, the first order necessary conditions for the parameters that minimize $L[E q$. (12)] require the solution of an eigenvalue problem:

$$
(G-\lambda I) a=0
$$

where the matrix $G$ is defined by:

$$
G_{i k}=A_{i k}-B_{i} B_{k}
$$

In reference 2, we did not include the constraint in the Lagrangian function (L). When the Lagrange multiplier $(\lambda)$ is zero, first order necessary conditions for the parameters that minimize $L$ [Eqs. (10) and (11)] are a set of $N+1$ homogeneous equations:

$$
D s=0
$$

where the column vector $s$ is defined by: 


$$
s=\left[\begin{array}{l}
d \\
a
\end{array}\right]
$$

and $D$ is a real symmetric matrix:

$$
D=\left[\begin{array}{ll}
1 & B \\
B^{T} & A
\end{array}\right]
$$

Eq. (15) will have a zero solution unless the matrix $D$ is singular and in general the matrix $D$ will not be singular. In reference 2, we estimated an approximate solution of Eq. (15) by adding a small pertubation to make the matrix $D$ singular and by solving the resultant eigenvalue problem:

$$
D s=\lambda s
$$

The solution of Eq. (18) that corresponds to the eigenvalue $\gamma$ with the smallest magnitude is our best approximate solution to Eq. (15).

Both our previous method and our current method require the solution of eigenvalue problems. However, the justification for the two methods are quite different. The first order necessary conditions for the parameters that minimize the constrained Lagrangian function require the solution of the first eigenvalue problem [Eq. (13)]. The first order necessary conditions for the parameters that minimize the unconstrained Lagrangian function require the solution of a set of $N+1$ homogeneous equations. The approximate solution of the set of homogeneous equations requires the solution of the second eigenvalue problem [Eq. (18)].

We conclude this section by showing that our current method satisfies the necessary conditions for a local minimum. The second partial derivative matrix of the Lagrangian function is called the Hessian matrix. Sufficient conditions for a local minimum are that the Hessian matrix is positive definite:

$$
Q=\sum_{i=0}^{N} \sum_{j=0}^{N} v_{i} v_{j} H_{i j}>0
$$


where $v$ is an arbitrary vector (with a positive magnitude) and the Hessian matrix is defined by:

$$
H_{i j}=\frac{\partial^{2} L}{\partial s_{l} \partial s_{j}}
$$

where $s_{0}=d$ and $s_{l}=a_{l}$ for $i=1$ to $N$.

For our Lagrangian function [Eq. (7)], the Hessian matrix is given by:

$$
\begin{gathered}
H_{00}=2 \\
H_{0,}=2 B_{i} \\
H_{i j}=2\left(A_{i j}-\lambda \delta_{i j}\right)
\end{gathered}
$$

where $\delta_{i j}$ is the Kronecker delta (the elements of the identity matrix).

The quadratic form for the Hessian matrix $(Q)$ is given by:

$$
\left.Q=2\left\{v_{0}\right)^{2}+v_{0} \sum_{i=1}^{N} v_{i} B_{i}+\sum_{i=1}^{N} \sum_{j=1}^{N} v_{i} v_{j} A_{i j}-\lambda \sum_{i=1}^{N}\left(v_{i}\right)^{2}\right\}
$$

We can modify the third term in Eq. (24) by using Eq. (14), the definition of the $G$ matrix:

$$
v^{T} A v=v^{T}\left(G+B B^{T}\right) v=v^{T} G v+\left(B^{T} v\right)^{2}
$$

To transform the quadratic form for the $G$ matrix, we will make a coordinate transformation. Let the $\psi_{n}$ be the eigenvectors for the $G$ matrix: 


$$
G \Psi_{n}=\lambda_{n} \Psi_{n}
$$

Since $G$ is a real symmetrical matrix, we can construct a set of eigenvectors that are an orthonormal basis for the space of $\mathrm{N}$ vectors. Let the columns of the matrix $T$ be the eigenvectors for the $G$ matrix:

$$
T=\left[\begin{array}{llll}
\Downarrow_{1} & \Psi_{2} & \ldots & \psi_{N}
\end{array}\right]
$$

$T$ is an orthogonal matrix. We will make a coordinate transformation from the $v$ vector to a new vector $(r)$ :

$$
v=\operatorname{Tr}
$$

Using our new coordinates, the quadratic form for the $\mathbf{G}$ matrix becomes:

$$
v^{T} G v=r^{T} T^{T} G T r
$$

Since the columns of the matrix $\mathbf{T}$ are the eigenvectors for the $\mathbf{G}$ matrix:

$$
G T=T E
$$

where:

$$
E=\operatorname{diag}\left[\lambda_{1}, \lambda_{2},, \lambda_{N}\right]
$$

Since $T$ is an orthogonal matrix, the right side of Eq. (29) becomes: 


$$
v^{T} G v=r^{T} E r=\sum_{i=1}^{N} \lambda_{i}\left(r_{i}\right)^{2}
$$

An orthogonal transformation preserves length:

$$
\sum_{i=1}^{N}\left(v_{i}\right)^{2}=v^{T} v=r^{T} T^{T} T r=r^{T_{r}}=\sum_{i=1}^{N}\left(r_{i}\right)^{2}
$$

Our goal is to show that the Hessian matrix is positive definite. We have modified several terms on the right side of Eq. (24). Using Eq. (25), Eq. (32), and Eq. (33), Eq. (24) becomes:

$$
\left.Q=2\left\{v_{0}+B^{T} v\right)^{2}+\sum_{i=1}^{N}\left(\lambda_{i}-\lambda\right)\left(r_{i}\right)^{2}\right\}
$$

The parameter $\lambda$ in Eq. (34) is one of the eigenvalues of $G$ [see Eq. (13)]. If $\lambda$ is the smallest of the eigenvalues, the second term in Eq. (34) will be non negative. The Hessian matrix is positive definite except for the following special case: assume $v_{0}=0$ and $\lambda_{1}$ is the smallest of the eigenvalues. Choose the $r$ vector with $r_{1}=1$ and the other $r_{i}=0$. Then the second term in Eq. (34) will be zero. The $v$ vector is determined by Eq. (28). The first term will be positive unless $B$ and $v$ are orthogonal $\left(B^{T_{v}}=0\right)$.

For our previous method, the solution of Eq. (18) that corresponds to the eigenvalue with the smallest magnitude is our best approximate solution to Eq. (15). For our current method, we chose the smallest of the eigenvalues. Thus, $\lambda$ can be negative and may not have the smallest magnitude. 


\section{NUMERICAL EXPERIMENTS}

We have developed a method for obtaining the last squares fit of a hyperplane to uncertain data. In this section, we will apply our method to synthetic data sets in three dimensions. We will compare the results for our eigenvalue method with three other methods: our previous method, Pratt's method, and a partial information approach [neglecting one equation at a time in Eq. (15)]. The partial information approach produces four different solutions for the unknown parameters, rather than a single solution. For each method, we can calculate an error measure [the error is the square root of the first term on the right side of Eq. (6)]. We will find that our method produces the smallest errors.

We consider a plane in three dimensional space with parameters:

$a=(0.57735,0.57735,0.57735)$ and $d=-1$. To create a synthetic data set, we calculate 81 data points with random errors that are proportional to a parameter $(\sigma)$. Given the data set and the weights $\left(w_{j}=1 / 81\right)$, we can calculate the $B$ vector and the $A$ matrix and solve the eigenvalue problem. The error measure is displayed in Table 1 for five values of $\sigma$ for the four methods (New, Old, Pratt, and Partial Information). In the last section, we proved that our new method should have the minimum error. In Table 1, our new method always has the lowest error, followed by: the old method, the Pratt method, and the partial information method. As the data errors increase ( $\sigma$ becomes larger), the errors for our new method become substantially less than the errors for the other methods (when there are no errors in the data, all four of the methods have zero errors).

The parameters $a$ and $d$ calculated by our new method are displayed in Table 2 for five values of $\sigma$. As $\sigma$ increases, the magnitude of the parameter $d$ increases. There is not a trend in the direction parameters $\left(a_{i}\right)$; sometimes they are greater than the true values and sometimes they are less. 
Table 1. The error measure for synthetic data set for four methods.

\begin{tabular}{|c|c|c|c|c|}
\hline Method & $\sigma=0.1$ & $\sigma=0.2$ & $\sigma=0.4$ & $\sigma=0.8$ \\
\hline New & 0.034953 & 0.070039 & 0.140247 & 0.279550 \\
\hline Old & 0.034966 & 0.070148 & 0.141092 & 0.285753 \\
\hline Pratt & 0.034968 & 0.070182 & 0.141465 & 0.287659 \\
\hline P1 & 0.035002 & 0.070398 & 0.142629 & 0.293255 \\
\hline P2 & 0.035030 & 0.070597 & 0.143843 & 0.297911 \\
\hline P3 & 0.035031 & 0.070608 & 0.143947 & 0.298470 \\
\hline P4 & 0.034983 & 0.070259 & 0.141706 & 0.287899 \\
\hline
\end{tabular}

Table 2. The least squares estimates of parameters $a$ and $d$ for five synthetic data sets.

\begin{tabular}{|c|c|c|c|c|}
\hline$\sigma$ & $a_{1}$ & $a_{2}$ & $a_{3}$ & $d$ \\
\hline 0.0 & 0.57735 & 0.57735 & 0.57735 & -1.00000 \\
\hline 0.1 & 0.57704 & 0.57576 & 0.57924 & -1.05197 \\
\hline 0.2 & 0.57733 & 0.57513 & 0.57958 & -1.10130 \\
\hline 0.4 & 0.57906 & 0.57591 & 0.57708 & -1.19361 \\
\hline 0.8 & 0.58441 & 0.58196 & 0.56550 & -1.36040 \\
\hline
\end{tabular}




\section{CONCLUSIONS}

We have developed a method for obtaining the least squares fit of a hyperplane to uncertain data. The first order necessary conditions require the solution of an eigenvalue problem. We have shown that the solution satisfies the second order conditions (the Hessian matrix is positive definite). Thus, our solution satisfies the sufficient conditions for a local minimum. We have performed numerical experiments that demonstrate that our solution is superior to alternative methods. 


\section{REFERENCES}

1. Lawson, C. L. and R. J. Hanson (1974). Solving Least Squares Problems, PrenticeHall, Englewood Cliffs, New Jersey.

2. Reister, D. B. and M. D. Morris (1994). "A Method for Obtaining a Least Squares Fit of a Hyperplane to Uncertain Data", ORNL/TM-12686, Oak Ridge National Laboratory.

3. Pratt, V. (1987). "Direct Least-Squares Fitting of Algebraic Surfaces" Computer Graphics, 21, 145-152.

4. Nievergelt, Y. (1994). "Total Least Squares: State-of-the-Art Regression in Numerical Analysis", SLAM Review, 36, 258-264. 


\section{INTERNAL DISTRIBUTION}

1-5. J. Barhen

6. M. Beckerman

7. C. W. Glover

8-12. W. C. Grimmell

13. J. P. Jones

14. H. E. Knee

15-19. R. C. Mann

20. M. D. Morris

21. E. M. Oblow

22-26. C. E. Oliver

27. L. E. Parker

28. N. S. V. Rao
29-33. D.B. Reister

34. S. Shekhar

35-39. R. F. Sincovec

40. E. C. Uberbacher

41. M. A. Unseren

42. CSMD Reports Office

43-44. Laboratory Records Department

45. Laboratory Records, ORNL-RC

46. Document Reference Section

47. Central Research Library

48. ORNL Paten Office

\section{EXTERNAL DISTRIBUTION}

49. Dr. Fred Aminzadeh, 401 Paseo Estrella, Anaheim Hills, CA 92807

50. Dr. John Baillieul, Aerospace and Mechanical Engineering Department, Boston University, 110 Cummington St., Boston, MA 02215

51. Dr. John Blair, JBX Technologies, 25 Moore Road, Wayland, MA 01778

52. Professor Roger W. Brockett, Harvard University, Pierce Hall, 29 Oxford St., Cambridge, MA 02138

53. Mr. Steve Holland, Robotics, B/MD-63, General Motors Corporation, NAO Manufacturing Center, 30300 Mound Rd., Warren, MI 48090-9040

54. Professor Takeo Kanade, Computer Science and Robotics, Carnegie Mellon University, Pittsburgh, PA 15213-3890

55. Dr. Oscar P. Manley, Division of Engineering, Mathematical, and Geosciences, Office of Basic Energy Sciences, ER-15, U.S. Department of Energy, Germantown, Washington, DC 20545

56. Dr. K. S. Narendra, Yale University, Center for Systems Science, Department of Electrical Engineering, P.O. Box 208267, New Haven, CT 06520-8267

57. Mr. Peter M. O'Donnell, Naval Air Engineering Center, Code 02T (POD), Lakehurst, NJ 08733-5000 
58. Mr. Steve Singer, NAWAIRWARCENACDIVLKE, Code 02T (SS), Bldg. 562 , Room 310, Lakehurst, NJ 08733-5000

59. Dr. Wes Snyder, Department of Radiology, Bowman Gray School of Medicine, N.C. Baptist Hospital School of Medicine, 300 S. Hawthorne Dr., WinstonSalem, NC 27103

60. Professor Mary F. Wheeler, Department of Mathematical Sciences, Rice University, P.O. Box 1892, Houston, TX 77251

61. Office of Assistant Manager for Energy Research and Development, U.S. Department of Energy, Oak Ridge Operations Office, P.O. Box 2001, Oak Ridge, TN 37831-8600

62-63. Office of Scientific \& Technical Information, P. O. Box 62, Oak Ridge, TN 37830 